\title{
Current and Future Treatment Options in Primary Achalasia. The Role of POEM
}

\author{
Lars Lundell
}

Division of Surgery,

Department of Clinical

Science, Intervention and Technology (CLINTEC), Karolinska Institutet, Stockholm, Sweden; Department of Surgery, Odense University Hospital, Odense, Denmark

\author{
Address for correspondence: \\ Lars Lundell MD PhD \\ Division of Surgery, \\ Department of Clinical \\ Science, Intervention and \\ Technology (CLINTEC), \\ Karolinska Institutet, \\ Stockholm, Sweden; \\ Department of Surgery, \\ Odense University Hospital, \\ Odense, Denmark \\ lars.lundell@ki.se
}

More than 300 years ago Thomas Willis (1621-1675) described the history of a 38-year-old man from Oxford who presented with an inability to swallow. Due to severity and long duration of symptoms he was desperate and starving. After serious considerations Willis came to the conclusion that the patient's problem was caused by lower esophageal narrowing leading to a massively dilated esophagus. The greatness of Sir Willis [1] was that he extended his ambition, beyond the establishment of the diagnosis, by designing a novel device to alleviate the underlying defect and thereby constructed the first dilator. This device consisted of a piece of whalebone with a sponge adapted to its distal end. The patient was instructed to insert the device transorally through the esophagus to achieve dilatation and push the swallowed food into the gastric reservoir for further digestion. This was apparently an effective therapy and the patient lived for many years without major complaints.

The mechanism behind the lower esophageal narrowing reported by Sir Willis remained unclear for a long period of time. With the landmark studies by Arthur Hurst it was possible to sort out causative factors. He had been acquainted with the use of barium to study gastrointestinal motility and noted that the lower esophagus failed to relax in these patients and thereby coined the term achalasia [2].

Achalasia is a Greek term meaning lack or absence of relaxation with particular implication of the lower esophageal sphincter (LES). The disease is defined as esophageal dysmotility, which is manometrically characterized by an incomplete relaxation of the LES. Frequently aperistalsis of the esophageal body is seen at the time of the first clinical manifestation of the disease or will progress during the course of the disease. Achalasia is a rare disease and estimates of its true incidence and prevalence are complicated by the fact that it may even be asymptomatic. Most studies addressing the epidemiology [3-5] have been retrospective and therefore it is understandable why the figures vary from 0.03 to 1.5 cases per 100,000 inhabitants and year, with no race and gender predilection. There seems to be two-incidence peaks. One minor peak is seen in the 15-30 age ranges and the predominant one in the seventh decade.

The normal swallow-induced peristaltic wave is propelled through the esophagus via excitatory and inhibitory neuronal mechanism transmitted via the enteric neuronal plexus and also external innervations via primarily the vagus nerve. These complex mechanisms regulate the muscular events that move the food bolus from the pharynx into the stomach and involve both cholinergic and peptidergic excitation, contraction and aboral relaxation ahead of the bolus in the respective muscle wall components. Inhibitory influences have generally been seen to be dependent on non-adrenergic, non-cholinergic transmission (NANC). A variety of mediators of NANC have been proposed such as: vasoactive intestinal peptide (VIP), ATP, and pituitary adenylate cyclase polypeptide. During recent years data have accumulated to show that nitric oxide (NO) may be the major inhibitory transmitter governing relaxation of esophagus and LES [6-10]. The interstitial cells of Cajal (ICC) represent another potential key factor, for the physiology as well as the pathophysiology. The interstitial cells of Cajal are abundant in the LES and may serve as pacemakers, as a generator of smooth muscle hyperpolarization, as a mechanical sensor and as a mediator of neurotransmission. The complete role of ICC in achalasia has, however, to be further researched. For instance, a reduced number of ICC have been reported in pediatric patients but also the absence of NOS positive enteric nerves surrounding the ICC. The early incidence peak 
has initiated studies in the pediatric achalasia population to determine eventual hereditary etiologic components. A consistent finding in most studies is the presence in the muscle tissue of different degrees of inflammation [11]. Another frequent finding is myenteric fibrosis and immunohistochemical studies have demonstrated the presence of lymphocyte infiltration and collagen deposition within the myenteric plexus. The majority of lymphocytes in these achalasia specimens are of T-lymphocyte character, exhibiting active cytotoxic characteristics. Corresponding observations offer indirect evidence for autoimmune pathogenetic mechanisms to be involved with or without the contribution from exogenous infections agents.

Based on the understanding and comprehension of the pathogenesis of idiopathic achalasia, it is understandable that there is little capacity, if any, for the regeneration or recovery of the abnormal or absence of peristalsis. Accordingly, no treatment is currently available to reverse the underlying disease specific processes. Consequently, even today, so many years after Sir Willis' milestone observations, the contemporary therapeutic aims are to alleviate symptoms by opening the LES. This can theoretically be accomplished by pharmacological means (including chemical-neuro paralysis), mechanical dilatation or by surgical myotomy and lately the endoscopic myotomy approach (POEM). Esophageal high resolution manometric pressure topography has allowed for the differentiation of achalasia into three subtypes with potential treatment outcome implications $[10,12]$. Type I achalasia is associated with absent peristalsis and minimal esophageal body pressurization. Type II achalasia is associated with panesophageal pressurization related to a compression effect. Type III achalasia has evidence of abnormal contractility (spastic). To date, many studies have shown that subtype II has the best prognosis, followed by subtype I; subtype III is more difficult to treat.

\section{THERAPEUTIC OPTIONS}

Drug induced LES relaxation can be induced by $\mathrm{Ca}^{2+}$ channel blockers, long-acting nitrates, $\beta 2$ - adrenergic agonists, anticholinergics and phosphodiesterase blockers $[9,10]$. Outcomes data on drug treatment vary significantly. It seems as if the most promising data have emerged from studies with sublingual nifedipine. Overall most pharmacological studies have observed that a drug related effect on LES nadir or basal pressure is at the very best only $50 \%$ of that reached by pneumatic dilation or surgical myotomy. It is therefore recommended that drug therapy should not be used as a primary treatment of idiopathic achalasia. Another pharmacological approach is represented by botulinum toxin, which blocks the pre-synaptic release of acetylcholine. This injection is followed by a reversible paralysis of the sphincter muscle $[13,14]$. Botulinum toxin injection into the LES should be reserved for those who cannot undergo definitive more effective therapies.

\section{Pneumatic dilatation}

The simple technique of forceful dilatation of the LES is still considered by many to represent first line therapy after the diagnosis has been established $[10,15-18]$. The objective of this therapy is to produce a controlled over stretching and tearing of the LES and thereby rendering it incompetent and breaking the vicious circle. Modern dilatation devices allow a safe and controlled inflation of a rigid balloon to a pressure of 10-12 Psi for a period varying from 60 second to 3 minutes. It has not been clarified whether the diameter of the balloon has to be $>30 \mathrm{~mm}$, or the most effective dilatation regimen, but a post procedure LES pressure of $<10 \mathrm{mmHg}$ is recommended, although not frequently practiced in daily routine activities. The short-term success rate varies from $60-90 \%$ and up to $90 \%$ success rate has been reported after repeated dilatations. The proportion of patients who need repeated dilatations range from $15-65 \%$. Although the early efficacy of pneumatic dilatations is indisputable, the long-term results are less encouraging and considered by some to be virtually unclear.

\section{Surgical myotomy}

It is now generally accepted that an anterior myotomy is the surgical procedure of choice in uncomplicated achalasia. To offer the patient adequate symptom control, the myotomy should not only divide the intra-abdominal portion of the sphincter but needs to be extended into the stomach as well, to divide the gastric-oblique fiber portion of the sphincter [19, 20]. Four group of patients have traditionally been considered good candidates for surgical myotomy. The first group is represented by younger patients ( $<40$ years) in whom lifelong dilatation is predictable. The second group includes those who do not respond adequately to conservative treatment (pneumatic dilatations). The third group consists of patients who are considered high risk for failure after conservative dilatation such as those having had previous surgery at the gastroesophageal junction, esophageal diverticula's or a distorted lower esophageal anatomy secondary to a tortuous or grossly dilated distal esophagus. Last but not least we have the group of patients who explicitly prefer surgery to dilatation [21]. As a result of successful surgical therapy for achalasia, a different disease, i.e. gastrointestinal reflux disease, is induced. Accordingly, a fundoplication has to be added to the myotomy and a partial fundic wrap is most often recommended. It can be argued whether an anterior (Dor) or a posterior wrap (Toupet) should be preferred [20].

\section{Surgical myotomy versus pneumatic dilatation}

None of the available achalasia treatments are curative, and around $20 \%$ of patients have symptoms that may require additional treatments within 5 years, even during close clinical surveillance [15]. Six-20\% of such patients may even face progressive esophageal dilation and reach an end-stage disease status. Accordingly, the long-term therapeutic effects of respective strategy have to be considered. Although surgical myotomy seems to be associated with an initially better response rate than a single pneumatic dilatation, a wide spread opinion is that a series of dilations offers a reasonable mid-to long-term alternative to surgery [21-24]. In fact, a randomized pivotal trial compared this type of graded dilatation strategy with laparoscopic surgical myotomy and found it to be non-inferior in efficacy [25]. In meta-analyses, remission rates were found to be higher at both 3 and 12 months (short-term) after surgical myotomy compared to 
pneumatic dilatations. However, it was impossible to detect any differences in remission rates in the period from 2 to 5 years after initiation of therapy. It has to be recognized that, due to incomplete data sets, at the later follow-up time points, the results have to be interpreted with caution. It has also to be pointed out that in the pivotal European trial, the per-protocol differences in success rates seemed to differ in favour of surgical myotomy, the longer these patients were followed [26]. In conclusion, it currently seems as if achalasia patients originally randomized to either laparoscopic surgical myotomy or repeated pneumatic dilatations as main therapeutic strategies are followed up for more than 10 years; surgical myotomy strengthens its superiority over the pneumatic dilatation strategy $[27,28]$.

\section{Peroral endoscopic myotomy}

Peroral endoscopic myotomy has quite recently been launched as an attractive endoscopic technique for the definitive treatment of achalasia [29]. The procedure involves endoscopic submucosal dissection with the creation of a submucosal plane using a forward-viewing endoscope to access the circular muscle fibres to complete the myotomy. The myotomy is usually about $6 \mathrm{~cm}$ into the esophagus and $2 \mathrm{~cm}$ below the squamocolumnar junction. Moreover, with the growth of experience the endoscopic myotomy becomes transmural and more radical. Inoue and co-workers [29] reported that POEM significantly reduced the dysphagia symptom score and LES pressure in all cases studied. Subsequent studies originating from other institutions, incorporating limited number of patients each, confirmed the high success rate (89-100\%), even in patients who had several previous pneumatic dilatations [30]. This has led to an increasing use and enthusiasm with POEM which has the potential to be an outpatient clinical care procedure. However, there are some caveats and uncertainties with POEM that need to be clarified. The true recurrence rate of dysphagia has to be better defined and objectively assessed and this has been reported to occur mostly 12-18 months after the intervention. It is noteworthy to mention that the follow up period of most POEM studies has been relatively short (mean 6 months) [31]. Another concern with POEM is the occurrence of gastroesophageal reflux with a reported, quite substantial incidence. Moreover, there are issues confined to the safety and feasibility of POEM when introduced and practiced in institutions outside those of the pioneers [32-41].

As a natural orifice approach, POEM has the potential for less morbidity, quicker recovery, better cosmesis, longer myotomy, and a spared need of dissecting the thoracic and abdominal esophagus. It is obvious why the POEM's safety profile and effectiveness relative to alternative interventions has been met by wide acceptance by physicians, patients, and payers. Further, the clinical outcomes of POEM must be compared to the traditional treatments for achalasia, namely endoscopic pneumatic dilatation and surgical myotomy.

Peroral endoscopic myotomy versus surgical myotomy or pneumatic dilatations

During recent years a number of systematic reviews and meta-analyses have been carried out [32-44]. In those, 28 studies (2 RCTs and 26 observational studies) have been included. Most comparative studies on POEM include surgical myotomy $(n=21)$, with a minority on POEM versus pneumatic dilatation $(n=8)$; one study included all three interventions. All were published after 2012, though data from surgical myotomy and pneumatic dilatation often were older. Moreover, the follow-up was often shorter for POEM. Except for two 4-year observational studies, POEM follow-up averaged $\leq 2$ years. There were only two pediatric studies, with one each comparing POEM with pneumatic dilatations, and with surgical myotomy. Otherwise, most studies predominantly included patients 4070 years old, with a few studies comprising younger adults. Most studies reported on the baseline achalasia subtype, the majority of which had either predominantly type II and/or type I achalasia. All studies had a small sample size. Quite recently two randomized controlled clinical trials compared POEM with surgical myotomy or pneumatic dilatations $[45,46]$.

Based on these analyses it can be concluded that POEM has similar outcomes to surgical myotomy and greater efficacy than pneumatic dilatations. Reflux remains a critical outcome after POEM with unknown long-term clinical significance-consequences due to insufficient long-term data and inconsistent reporting.

Based on the available evidence it can therefore be concluded that adult and pediatric patients with type I and II achalasia can be treated with either POEM or laparoscopic surgical myotomy based on the surgeon and patient's shared decision-making. Based on limited experience, it seems as if POEM can be recommended over laparoscopic surgical myotomy for the subtype III in adult or in pediatric achalasia.

Based on available clinical evidence POEM can be recommended over PD in all patients with achalasia.

\section{ASPECTS ON FUTURE DEVELOPMENTS}

There are a number of remaining questions relevant to the current and future treatment and understanding of achalasia, which urgently require focus and research priorities within the academic-medical community:

- Since achalasia is such a rare disease, a centralization of these cases within individual countries is recommended, to enhance and facilitate therapeutic outcomes as well as research opportunities. In this issue of the Journal of Gastrointestinal and Liver Diseases, Tefas et al. [47] reported the Romanian experience of POEM, one of the few studies from Eastern Europe. They found a high success rate and a low rate of adverse events in short, medium and long-term in 136 patients with achalasia treated with POEM.

- Achalasia subtype III-specific outcomes after POEM vs. surgical myotomy are needed.

- More research is required on pediatric populations regardless of the subtype of the disease.

- Longer term results are required for all outcomes given the chronic nature of achalasia. Surveillance and follow-up past 10 years is necessary.

- Better instruments, to determine the presence of dysphagia rather than the Eckardt score, should be developed to be used in addition to more accurate, objective outcome alternatives i.e. high-resolution manometry and timed barium swallow. 
- Studies are required to establish whether there is a correlation between post-POEM LES pressure and post-POEM outcomes.

- Evidence suggests that POEM leads to greater postoperative reflux. However, data are required on the role, patient acceptance, and efficacy of PPI use after POEM. Studies have to be prioritized over strategies to address undesirable effects for both POEM and surgical myotomy.

- Long term follow-up measures are warranted to determine the incidence and severity of esophagitis after POEM, as well as the incidence of sequelae of esophagitis.

Conflicts of interest: None to declare.

\section{REFERENCES}

1. Willis T. Pharmaceutice rationalis sive diatriba do medicamentorum operationibus in humano corpore. Hagae-Comitis: Leers, 1674.

2. Hurst AF, Rake GW. Achalasia of the cardia. Q J Med 1930:23:491-507.

3. Mayberry JF. Epidemiology and demographics of achalasia. Gastrointest Endoscopy Clin N Am 2001;11:235-248.

4. Sonneberg A, Massey BT, McCarthy DJ, Jacobsen SJ. Epidemiology of hospitalisation for achalasia in the United States. Dig Dis Sci 1993;38:233-244. doi:10.1007/BF01307540

5. O’Neill OM, Johnston BT, Coleman HG. Achalasia: a review of clinical diagnosis, epidemiology, treatment and outcomes. World J Gastroenterol 2013;19:5806-5812. doi:10.3748/wjg.v19.i35.5806

6. Sanders KM, Ward SM. Nitric oxide as a mediator of nonadrenergicnoncholinergic neurotransmission. Am J Physiol 1992;262:G379-G392. doi:10.1152/ajpgi.1992.262.3.G379

7. Park W, Vaezi MF. Etiology and pathogenesis of achalasia: the current understanding. Am J Gastroenterology 2005;100:1404-1414. doi:10.1111/j.1572-0241.2005.41775.x

8. Kraichely RE, Farrugia G. Achalasia: physiology and etiopathogenesis. Dis Esophagus 2006;19:213-223. doi:10.1111/j.1442-2050.2006.00569.x

9. Ates F, Vaezi MF. The Pathogenesis and Management of Achalasia: Current Status and Future Directions. Gut Liver 2015;9:449-463. doi:10.5009/gnl14446

10. Boeckxstaens GE, Zaninotto G, Richter JE. Achalasia. Lancet 2014;383:83-93. doi:10.1016/S0140-6736(13)60651-0

11. Döhla M, Leichauer K, Gockel I, et al. Characterization of esophageal inflammation in patients with achalasia. A retrospective immunohistochemical study. Hum Pathol 2019;85:228-234. doi:10.1016/j.humpath.2018.11.006

12. Ihara E, Muta K, Fukaura K, Nakamura K. Diagnosis and Treatment Strategy of Achalasia Subtypes and Esophagogastric Junction Outflow Obstruction Based on High-Resolution Manometry. Digestion 2017;95:29-35. doi:10.1159/000452354

13. Michael J, Fazel A, Montazeri G, Yaghoobi M, Malekzadeh R. Randomized trial comparing botulinus toxin injection to pneumatic dilatation for the treatment of achalasia. Aliment Pharmacol Ther 2001;15:1389-1396. doi:10.1046/j.1365-2036.2001.01065.x

14. Bansal R, Nostrand TT, Scheiman JM, et al. Intrasphincteric botulinus toxin versus pneumatic dilation for treatment of primary achalasia. J Clin Gastroenterol 2003;36:209-214. doi:10.1097/00004836-200303000-00005

15. Vantrappen G, Hellemans J, Deloof W, Valembois P, Vandenbroucke J. Treatment of achalasia with pneumatic dilatations. Gut 1971;12:268275. doi:10.1136/gut.12.4.268
16. Gideon RM, Castell DO, Yarze J. Prospective randomized comparison of pneumatic dilatation technique in patients with idiopathic achalasia. Dig Dis Sci 1999;9:1853-1857. doi:10.1023/a:1018898824135

17. Mikaeli J, Bishehsari F, Montazeri G, Yaghoobi M, Malekzadeh R. Pneumatic balloon dilatation in achalasia: a prospective comparison of safety and efficacy with different balloon diameters. Aliment. Pharamacol Ther 2004;20:431-436. doi:10.1111/j.1365-2036.2004.02080.x

18. Khan AA, Shah SWH, Alam A, Butt AK, Shafqat F. Sixteen years follow up of achalasia: a prospective study of graded dilatation using Rigiflex balloon. Dis Esophagus 2005;18:41-45. doi:10.1111/j.14422050.2005.00448.x

19. Andreollo NA, Earlam RJ. Hellers myotomy for achalasia: is an added antireflux procedure necessary? Br J Surg 1987;774:765-769. doi:10.1002/bjs.1800740903

20. Kumagai K, Kjellin A, Tsai JA, et al. Toupet versus Dor as a procedure to prevent reflux after cardiomyotomy for achalasia: results of a randomised clinical trial. Int J Surg 2014;12:673-680. doi:10.1016/j. ijsu.2014.05.077

21. Abir F, Modlin I, Kidd M, Bell R. Surgical treatment of achalasia: current status and controversies. Dig Surg 2004;21:165-176. doi:10.1159/000079341

22. Weber CE, Davis CS, Kramer HJ, Gibbs JT, Robles L, Fisichella PM. Medium and long-term outcomes after pneumatic dilation or laparoscopic Heller myotomy for achalasia: a meta-analysis. Surg Laparosc Endosc Percutan Tech 2012;22:289-296. doi:10.1097/ SLE.0b013e31825a2478

23. Yaghoobi M, Mayrand S, Martel M, Roshan-Afshar I, Bijarchi R, Barkun A. Laparoscopic Heller's myotomy versus pneumatic dilation in the treatment of idiopathic achalasia: a meta-analysis of randomized, controlled trials. Gastrointest Endosc 2013;78:468-475. doi:10.1016/j. gie.2013.03.1335

24. Cheng JW, Li Y, Xing WO, Lv HW, Wang HR. Laparoscopic Heller myotomy is not superior to pneumatic dilation in the management of primary achalasia Conclusions of a systematic review and meta-analysis of randomized controlled trials. Medicine (Baltimore) 2017;96:e5525. doi:10.1097/MD.0000000000005525

25. Boeckxstaens GE, Annese V, des Varannes SB, et al. Pneumatic dilation versus laparoscopic Heller's myotomy for idiopathic achalasia. N Engl J Med 2011;364:1807-1816. doi:10.1056/ NEJMoa1010502

26. Moonen A, Annese V, Belmans A, et al. Long-term results of the European achalasia trial: a multicentre randomised controlled trial comparing pneumatic dilation versus laparoscopic Heller myotomy. Gut 2016;65:732-739. doi:10.1136/gutjnl-2015-310602

27. Persson J, Johnsson E, Kostic S, Lundell L, Smedh U. Treatment of achalasia with laparoscopic myotomy or pneumatic dilatation: long-term results of a prospective, randomized study. World J Surg 2015;39:713-720. doi:10.1007/s00268-014-2869-4

28. Sediqi E, Tsoposidis A, Wallenius V, et al. Laparoscopic Heller myotomy or pneumatic dilatation in achalasia: results of a prospective, randomized study with at least a decade of follow-up. Surg Endosc 2020 Apr 17. doi:10.1007/s00464-020-07541-4

29. Inoue $H$, Minami $H$, Kobayashi $Y$, et al. Peroral endoscopic myotomy (POEM) for esophageal achalasia. Endoscopy 2010;42:265-271. doi:10.1055/s-0029-1244080

30. Kumagai K, Tsai JA, Thorell A, Lundell L, Håkanson B. Per-oral endoscopic myotomy for achalasia. Are results comparable to laparoscopic Heller myotomy? Scand J Gastroenterol 2015;50:505-512. doi:10.3109/00365521.2014.934915 
31. Parsa N, Khashab MA. POEM in the Treatment of Esophageal Disorders. Curr Treat Options Gastroenterol 2018;16:27-40. doi:10.1007/s11938018-0168-0

32. Marano L, Pallabazzer G, Solito B, et al. Surgery or Peroral Esophageal Myotomy for Achalasia: A Systematic Review and Meta-Analysis. Medicine (Baltimore) 2016;95:e3001. doi:10.1097/ MD.0000000000003001

33. Mu D, Li YY, Zhang MM, Zhang Y, Li Z, Li YQ. POEM for special patient cohorts: A review. J Dig Dis 2017;18:265-272. doi:10.1111/17512980.12473

34. Pandolfino JE, Gawron AJ. Achalasia: a systematic review. JAMA 2015;313:1841-1852. doi:10.1001/jama.2015.2996

35. Patel K, Abbassi-Ghadi N, Markar S, Kumar S, Jethwa P, Zaninotto G Peroral endoscopic myotomy for the treatment of esophageal achalasia: systematic review and pooled analysis. Dis Esophagus 2016;29:807-819. doi: $10.1111 /$ dote. 12387

36. Ramchandani M, Nageshwar Reddy D, Nabi Z, et al. Management of achalasia cardia: Expert consensus statements. J Gastroenterol Hepatol 2018;33:1436-1444. doi:10.1111/jgh.14097

37. Repici A, Fuccio L, Maselli R, et al. GERD after per-oral endoscopic myotomy as compared with Heller's myotomy with fundoplication: a systematic review with meta-analysis. Gastrointest Endosc 2018;87:934943.e18. doi:10.1016/j.gie.2017.10.022

38. Talukdar R, Inoue H, Nageshwar Reddy D. Efficacy of perora endoscopic myotomy (POEM) in the treatment of achalasia: a systematic review and meta-analysis. Surg Endosc 2015;29:3030-3046. doi:10.1007/ s00464-014-4040-6

39. Wei M, Yang T, Yang X, Wang Z, Zhou Z. Peroral esophageal myotomy versus laparoscopic Heller's myotomy for achalasia: a meta-analysis.
J Laparoendosc Adv Surg Tech A 2015;25:123-129. doi:10.1089/ lap.2014.0454

40. Zhang Y, Wang H, Chen X, et al. Per-Oral Endoscopic Myotomy Versus Laparoscopic Heller Myotomy for Achalasia: A Meta-Analysis of Nonrandomized Comparative Studies. Medicine (Baltimore) 2016;95:e2736. doi:10.1097/MD.0000000000002736

41. Oude Nijhuis RAB, Zaninotto G, Roman S, et al. European guidelines on achalasia: United European Gastroenterology and European Society of Neurogastroenterology and Motility recommendations. United European Gastroenterol J 2020;8:13-33. doi: $10.1177 / 2050640620903213$

42. Zaninotto G, Bennett C, Boeckxstaens G, et al. The 2018 ISDE achalasia guidelines. Dis Esophagus 2018;31:doy071. doi:10.1093/dote/doy071

43. Jung HK, Hong SJ, Lee OY, et al. 2019 Seoul Consensus on Esophagea Achalasia Guidelines. J Neurogastroenterol Motil 2020;26:180-203. doi:10.5056/jnm20014

44. Khashab MA, Vela MF, Thosani N, et al. ASGE guideline on the management of achalasia. Gastrointest Endosc 2020;91:213-227.e6. doi:10.1016/j.gie.2019.04.231

45. Werner YB, Hakanson B, Martinek J, et al. Endoscopic or Surgical Myotomy in Patients with Idiopathic Achalasia. N Engl J Med 2019;381:2219-2229. doi:10.1056/NEJMoa1905380

46. Ponds FA, Fockens P, Lei A, et al. Effect of Peroral Endoscopic Myotomy vs Pneumatic Dilation on Symptom Severity and Treatment Outcomes Among Treatment-Naive Patients With Achalasia: A Randomized Clinical Trial. JAMA 2019;322:134-144. doi:10.1001/jama.2019.8859

47. Tefas C, Boros C, Ciobanu L, Surdea-Blaga T, Tantau A, Tantau M. POEM: 5 Years of Experience in a Single East European Center. J Gastrointestin Liver Dis 2020;20:313-328. doi:10.15403/jgld-2676 